\title{
Total Ankle Replacement: Indications, Rehabilitation and Results
}

\author{
Marco Massobrio ${ }^{1}$, Giovanni Pellicanò ${ }^{1}$, Valter Santilli ${ }^{2}$ and Lucrezia Tognolo ${ }^{2 *}$ \\ ${ }^{1}$ Department of Orthopaedics and Traumatology, Sapienza University of Rome, Italy \\ ${ }^{2}$ Physical Medicine and Rehabilitation Division, Umberto I Hospital, Sapienza University of Rome, Italy
}

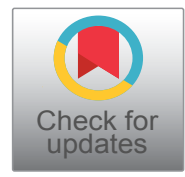

*Corresponding author: Lucrezia Tognolo, Physical Medicine and Rehabilitation Division, Umberto I Hospital, Sapienza University of Rome, Italy, E-mail: lucreziatognolo86@gmail.com

\section{Introduction}

Even though fusion is the main form of treatment for end-stage osteoarthritis (OA) of the ankle, total ankle replacement (TAR) is increasingly being recognized as an effective alternative procedure, with favorable clinical results and improved quality of life [1]. However, the results have been disappointing compared to those of hip and knee arthroplasty. In fact, the ankle is a more complex joint and its biomechanics and kinematics are strongly influenced by neighboring joints [2]. The ankle joint complex is made up of the talocalcaneal (subtalar), tibiotalar (talocrural), transverse-tarsal (talocalcaneonavicular) and inferior tibiofibular joint. This joints work together for the motion of the ankle: The key movement are plantar- and dorsiflexion (sagittal plane); ab-/adduction (transverse plane) and inversion-eversion (frontal plane). Three-dimensional motions (supination and pronation) are permitted by the combinations of these movements, through the ankle joints complex. This complex bears a force of approximately five times body weight during stance in normal walking, and up to thirteen times body weight during activities such as running. Static structures, as bony architecture and multiple different axes of joint motions partecipate to create most of the support and balance in standing. During quiet, level standing, the center of gravity projects approximately $5 \mathrm{~cm}$ anterior to the axis of the ankle joint. Soft tissues surrounding the joints of the ankle complex, expecially tendons, make understanding moment arms vital to comprehending the functioning of the joints as well as the impact of altering this balance.

A major difficulty in the selection of patients for the tibio-talar (TT) prosthesis consists in the clinical evaluation. The differential diagnosis for chronic ankle pain is quite broad. Ankle pain can be caused by intra-articular (TT, subtalar or midfoot joint) or extraarticular pathology and may be a result of a traumatic or nontraumatic event [3]. Primary osteoarthritis can significantly involve the TT joint, while both the subastragalic (SA) and the midfoot joints can also be affected. On the other hand, posttraumatic TT arthritis shows less differential diagnostic problems because of the clear role of articular changes and effects due to the trauma [4].

During the postoperative assessment it is important to evaluate not only changes occurred in the TT joint, but also those of the neighboring joints. The management of rehabilitation needs, after undergoing TAR, must be tailored to the individual patient because of the large number of preoperative, intraoperative and postoperative factors, which can influence the outcome [5].

Rehabilitation represents an important step to help patients recover from ankle surgery as quickly as possible and optimize the desired outcomes. Currently few works take into account the management of postsurgical rehabilitation, while many studies on the prosthetic ankle procedures are available in the literature [5-9]. Since there are no clear guidelines for rehabilitation protocols after ankle arthroplasty, the aim of the study is to present a postoperative physical therapy protocol. Moreover, the authors want to investigate changes in ankle range of motion (ROM) and discuss the indications and outcomes for TAR procedure.

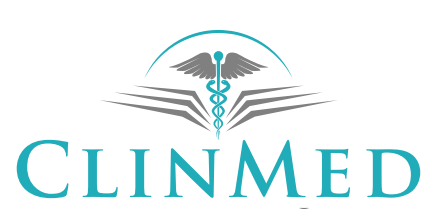

INTERNATIONAL LIBRARY

Citation: Massobrio M, Pellicanò G, Santilli V, Tognolo L (2018) Total Ankle Replacement: Indications, Rehabilitation and Results. Int J Foot Ankle 2:019.

Accepted: November 20, 2018; Published: November 22, 2018

Copyright: ( 2018 Massobrio M, et al. This is an open-access article distributed under the terms of the Creative Commons Attribution License, which permits unrestricted use, distribution, and reproduction in any medium, provided the original author and source are credited. 


\section{Materials and Methods}

\section{Patient demographics and study characteristics}

We conducted a retrospective study about 21 total ankle arthroplasties in 21 patients $(12$ men and 9 women) with the Mobility implant (DePuy Orthopaedics Inc), performed between June 2008 and April 2015. The average age of the patients was 62.4 years (range 48 to 64 years).

Ankles were classified according to the Canadian Orthopaedic Foot and Ankle Society (COFAS) classification system for end-stage ankle arthritis [10]. Patients were included if they were between the ages of 40 and 80 -years-old, had a body mass index (BMI) less or equal to 30 , had a low impact activity demand and had minimal osteopenia. We selected only patients with type 1 COFAS osteoarthritis (isolated ankle arthritis), ankle pain and decreased function not responsive to conservative treatment, functional limitation, good bone quality (able to support the prosthetic implant), ankle talus deformity not exceeding $10^{\circ}$ (tibio-talar tilt), muscular and tendinous validity (no neuromuscular deficits, flaccidity or spasticity) (Figure 1). The diagnosis was posttraumatic arthritis in 9 ankles and primary osteoarthritis in 12 patients [11].

Patients were not considered for total ankle arthroplasty if they were diagnosed with peripheral artery and metabolic diseases, obesity (BMI > 30), capsuloligamentous instability, and severe deformities at the knee, hindfoot, or forefoot.

All patients were assessed with clinical and radiographic follow-ups at 6 weeks, 3 months, 6 months and 1 year and then every 1 year thereafter. The mean follow-up for all 21 TARs was 28 months (range 12 to 60 months).

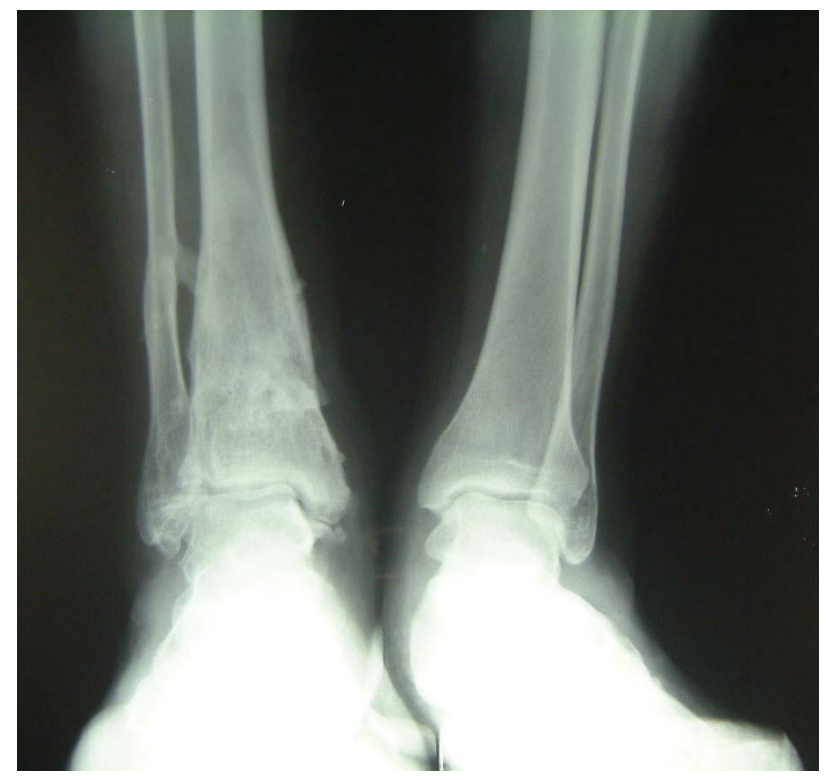

Figure 1: Comparative radiographic anteroposterior (AP) weight bearing assessment of isolated right ankle post-traumatic osteoarthritis, without deformity, with good bone quality.
All participants provided written informed consent and the Anatomical, Histological, Medico-Legal and Locomotor System Sciences Department Board of "La Sapienza" University of Rome approved the study.

\section{Surgical technique}

The Mobility Total Ankle System is a third generation, unconstrained, three-component mobile bearing ankle prosthesis. It consists of a tibial component, a talar component, and a mobile-bearing with a highly crosslinked polyethylene inlay. The deep central longitudinal sulcus on the talar component stabilizes the polyethylene insert and reduces the risk of bearing dislocations and subsidence. This feature justifies the smaller size of the prosthesis. The short tibial conical intramedullary stem provides primary fixation into the tibia, while stability of the talar component is enhanced by two pegs on the non-articulating aspect of the implant. The prosthesis is unconstrained, and the porous coating provides a surface for bone ingrowth (secondary stability).

The surgical procedure began with a midline anterior approach through a $10-15 \mathrm{~cm}$ incision over the middle part of tibio-talar joint. The incision was carried down deep between the tendons of anterior tibialis and extensor alluci longus muscles. The tibia and talus were prepared using bone cutting jigs to allow accurate bone resection of the distal tibial and proximal talar articular surfaces. Trial components were inserted, and the correct fit and alignment were verified by using fluoroscopic imaging, before the definitive components were implanted. Patients were usually discharged on the third or fourth postoperative day.

\section{Rehabilitation}

The rehabilitation program was performed on the following criteria: Pain relief, swelling reduction, restoration of full range of motion, muscles strengthening, restoration of balance and proprioceptive exercises until an optimal gait re-education.

During the first postoperative $24-48$ hours patients were educated to maintain the supine position with leg elevation to reduce swelling. A cast was applied during the first 2 weeks, to avoid weight bearing, with the ankle at $90^{\circ}$ to avoid skin tension caused by early mobilization and to allow complete wound healing.

Patients were educated to perform isometric exercises in order to prevent thromboembolic events. Active mobilization of the limb with the help of the therapist includes transfers from the supine to the sitting and standing position. Partial weight bearing with two Canadian crutches was commenced after 2 week, progressing to full weight bearing, as tolerated. The cast was maintained even during the night for 6 weeks to prevent the retraction of the Achilles tendon that could favor the attitude equinus of the foot. Partial weight-bearing was delayed by 2 weeks in patients 


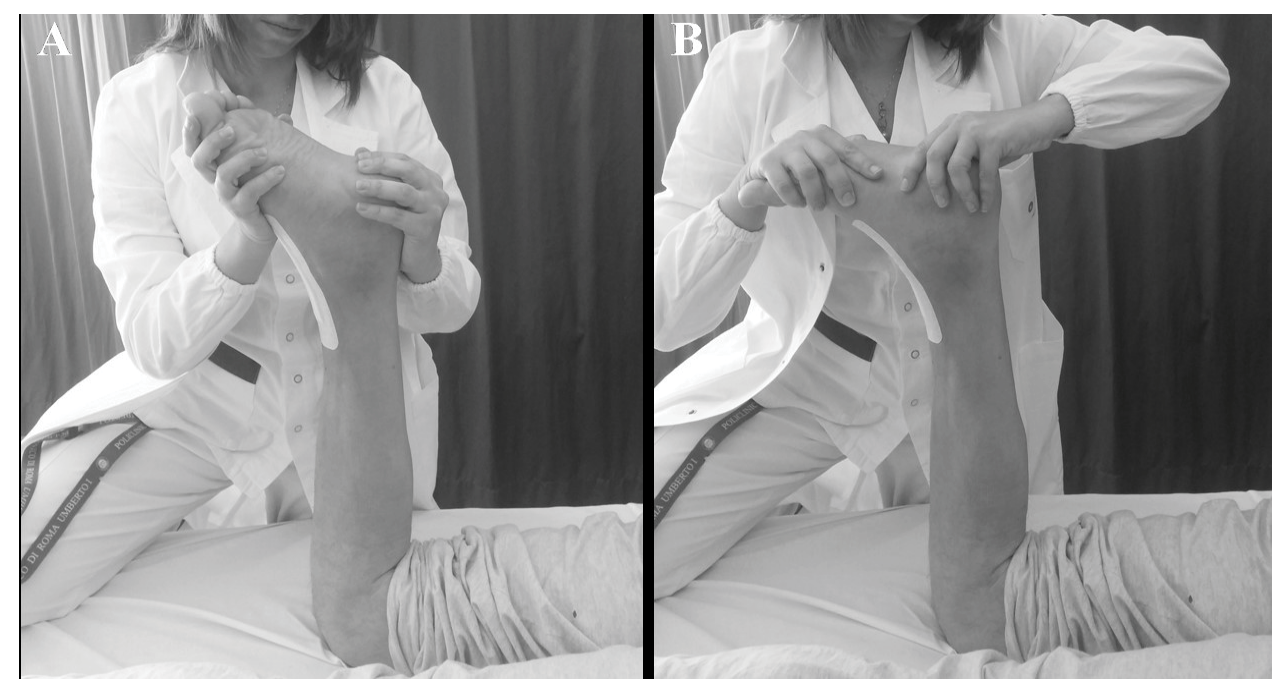

Figure 2: Alternating pressure on the heel $(A)$ and midfoot $(B)$ in prone position and knee flexed at $90^{\circ}$

who underwent surgical concomitant procedures and corrective osteotomy. The cast was definitively removed after 6 weeks.

During the first 6 weeks the cast has been removed during the physioterapic session. During this phase, only movements in the sagittal plane were allowed; dorsiflexion and plantar flexion of ankle and toes. At 2 weeks, progressive resistance exercises, as tolerated, were allowed to initiate.

At 3 weeks, the use of exercise bike without resistance was introduced in order to regain and improve the range of motion and to ensure a good aerobic training.

For the swelling management during all the phases of rehabilitation program, a lymph-draining massage was performed.

Active and assisted rehabilitation was performed with patients lying either prone or supine. The prone position with knee flexed to $90^{\circ}$ allowed to release posterior structures (muscles and tendons), permitting the therapist to work with less resistance. Alternating pressure on the heel and midfoot allows to regain the flexion-extension of the ankle (Figure 2).

At 4 weeks, open chain isotonic exercises were initiated if the incision had sufficiently healed. Strengthening exercises were performed alternating concentric and eccentric contractions of the ankle flexors and extensors.

After 6 weeks, patients were instructed to perform closed chain exercises with repeated raising on the tips, and alternatively walking on toes and heels. During this phase first passive and then active inversion and eversion were allowed.

Proprioceptive exercises were performed with patients sitting on a chair and rolling a ball under the arch of the foot, progressively regaining the flexionextension movement of the knee and ankle.
When full weight bearing was allowed, proprioceptive platform training was initiated to rescue the full plantar proprioception and to improve elasticity of the capsular ligamentous structures.

After 6 months, patients were allowed to gradually return to low impact sport activities.

\section{Clinical and radiographic assessment}

Clinical results were assessed using the American Orthopaedic Foot \& Ankle Society (AOFAS) hindfoot score [12]. The questionnaire is composed of nine items, distributed over three categories for a total of 100 points: pain (40 points), functional aspects (50 points), and alignment (10 points).

The radiographic assessment was based on anteroposterior (AP) and lateral weight bearing radiographs of the foot and ankle, as well as on lateral radiograph taken with the ankle in maximum plantar flexion and dorsiflexion. The Coetzee method allowed to determine the true ROM of the tibiotalar joint after total ankle arthroplasty [13] (Figure 3).

To assess bony integration, we used the method by Wood, et al. [14]. The authors considered that bony integration in any particular zone of the talar and tibial component had failed if there was a lucent line along the whole length of the zone or if the width of the lucency was $>2 \mathrm{~mm}$ at any point [15]. The radiographic assessment considered the presence of lucency areas and cystic radiolucencies (less common in patients with post-traumatic arthritis).

Radiographic evaluations were scheduled at 6 weeks, 3 months, 6 months and 1 year postoperatively and then every year. AOFAS score was measured preoperatively and postoperatively at 3 and 6 months of followup. Mean follow-up duration was 28 months, with a minimum of 18 months and a maximum of 5 years.

At the final follow-up, patients were asked whether 

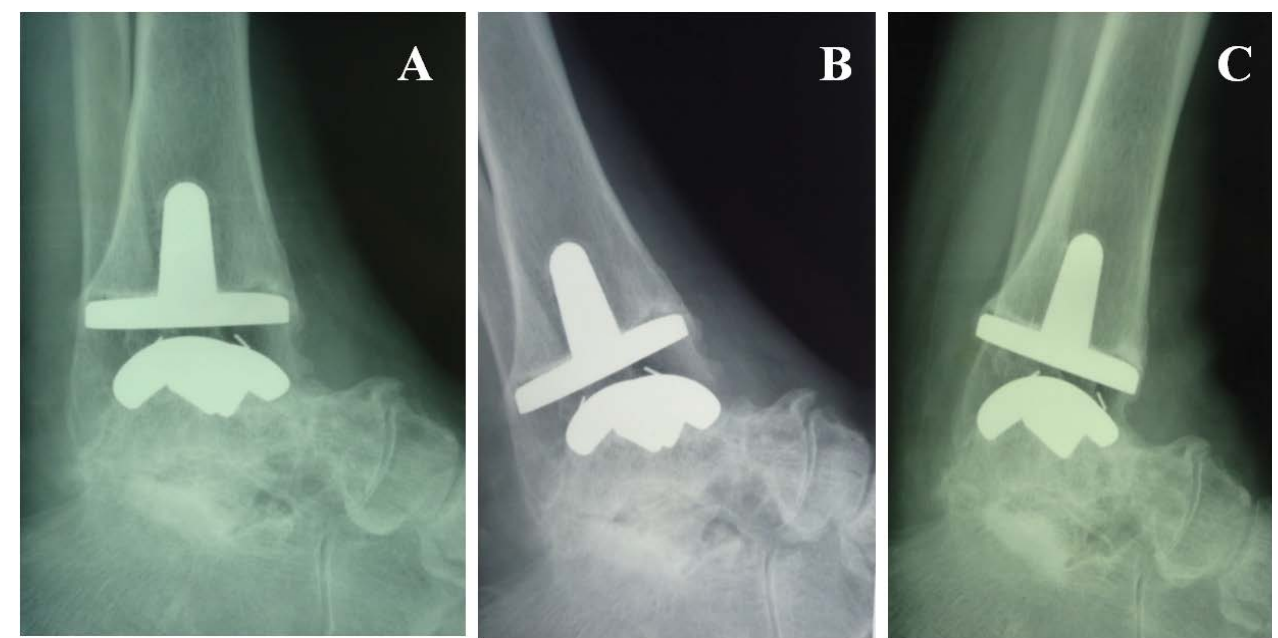

Figure 3: Radiographic lateral weight bearing assessment. A) Neutral position; B) Maximum plantar flexion; C) Maximum dorsiflexion.
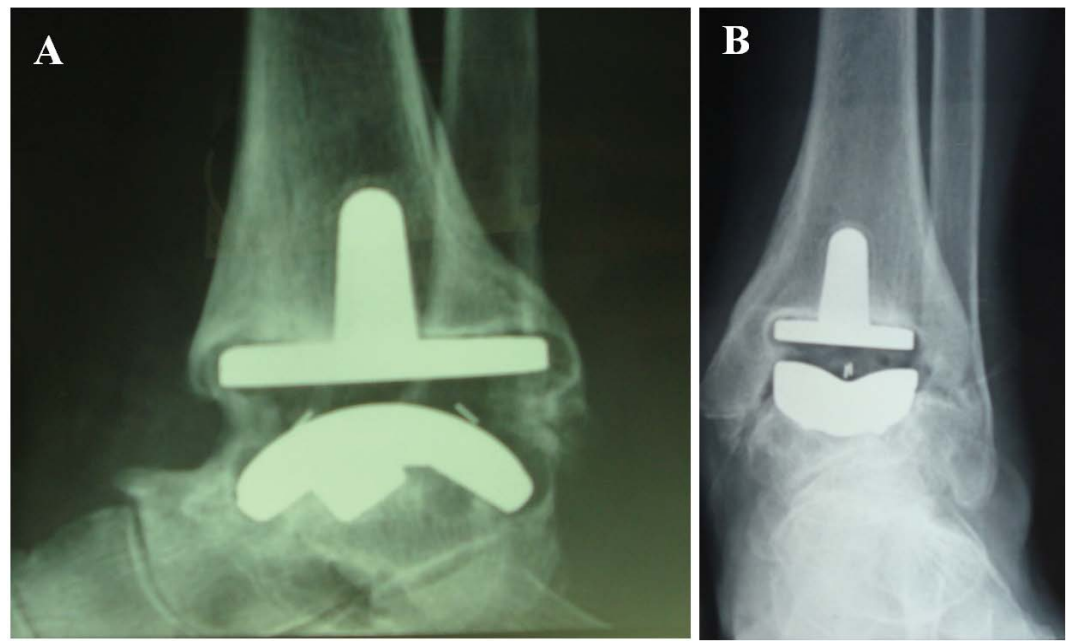

Figure 4: Radiographs showing subsidence of the talar component and radiolucency around the talar component. A) Lateral view; B) AP view.

they were satisfied (total, partial or no satisfaction) with the outcome of their TAR and if they would choose to have the operation again under similar circumstances.

\section{Statistical methods}

Statistical analysis of the data was done using paired Student's t test (significant $p<0.05$ ) and the statistical correlation coefficient for the correlation of two groups (clinical and radiographic assessment).

\section{Results}

\section{Clinical results}

The AOFAS score improved from a mean value of $41.6 \pm 8.6$ (range $34-58$ ) preoperatively to $84.8 \pm 5.6$ (range 73-93) at the 1-year follow-up. We observed best improvement in patients with post-traumatic arthritis (mean value $88.4 \pm 4.77$ ) than in patients with primary osteoarthritis (mean value $83 \pm 5.3$ ). In 2 patients with a longer follow-up we administered the AOFAS after 2 years, with the same results.

At the 1-year follow-up, 5 patients reported residual pain ( 1 patients at the subtalar joint, 2 at the medial malleolus, and 1 at the lateral malleolus). A deficit in dorsiflexion was observed in 2 patients. Dorsiflexion deficits were most likely due to the stiffness of posterior soft tissues, particularly the Achilles tendon.

11 patients reported that they were "very satisfied" with the surgery, 6 were "satisfied", and 4 were "partially satisfied" because of a restriction in plantar flexion due to hypertrophy of the surgical scar. All patients stated that they would undergo the same procedure again.

\section{Radiographic results}

The mean total range of ankle motion, assessed with the Coetzee method [14], improved from a mean of $21^{\circ}$ $\pm 7.5^{\circ}$ preoperatively $\left(\right.$ range $5^{\circ}$ to $3^{\circ}$ ) to a mean of $31.7^{\circ}$ $\pm 9.88^{\circ}$ (range 10 to $50^{\circ}$ ) postoperatively at the 1 -year follow-up, without statistical significance $(p>0.05)$.

There were no ankles with a significant varus/valgus deformity in one of the two components. $93.4 \%$ of the arthroplasty components were correctly centered in the frontal plane, and $86.7 \%$ were correctly centered in 

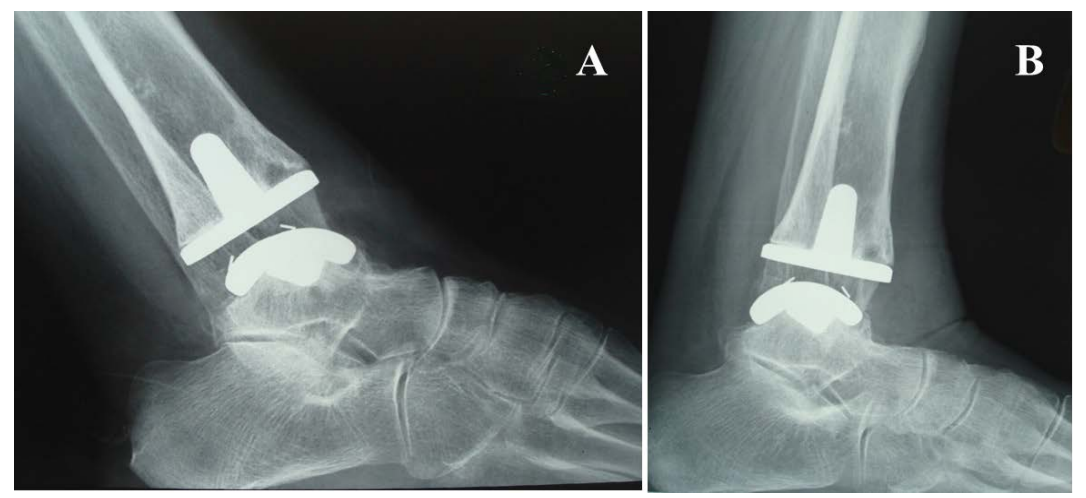

Figure 5: Radiographs at the 2-year follow up showing a periprosthetic asymptomatic cyst in the tibial component. No mobilization, no talar component subsidence. A) Maximum plantar flexion; B) Maximum dorsiflexion.

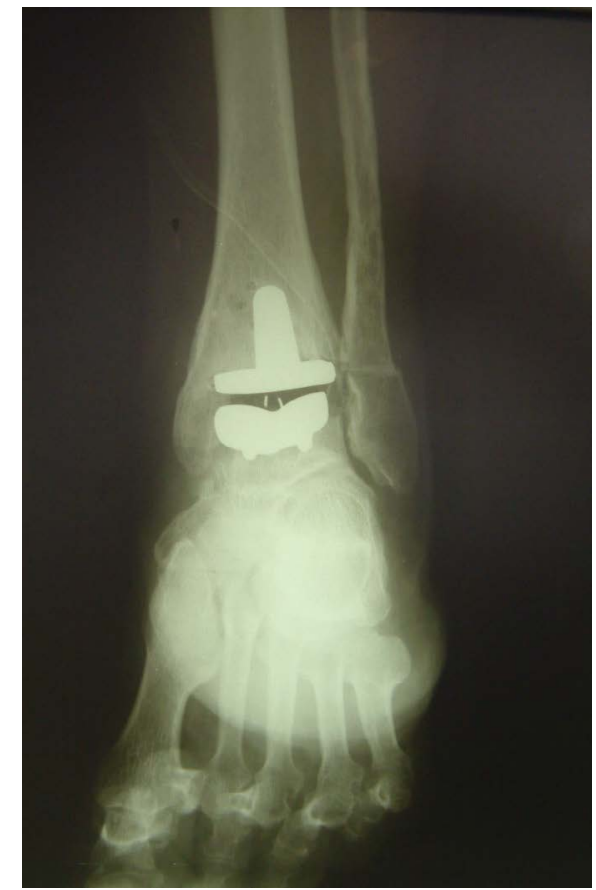

Figure 6: Stable fracture of the lateral malleolus. Conservative treatment.

the sagittal plane. The tibial components were placed in a mean of $2.1^{\circ} \pm 2.9^{\circ}$ (range $-3.1^{\circ}$ to $+5.4^{\circ}$ ) of varus relative to the tibial axis in the frontal plane ( $\alpha$ angle). The mean posterior slope was $6^{\circ} \pm 3.8^{\circ}$ (range $-5.8^{\circ}$ to $15.3^{\circ}$ ) relative to the tibia in the sagittal plane ( $\beta$ angle).

Lucencies were not seen in any of the zones around the talar component. Radiographs showed subsidence of the talar component $<5 \mathrm{~mm}$ in 3 ankles and a subsidence between 5 and $10 \mathrm{~mm}$ in 2 ankles (Figure 4). None of these 5 patients were associated with a clinical correlation in the radiographic finding. There were no cases of tibial component mobilization and migration. A periprosthetic asymptomatic cyst was detected in 1 patient with posttraumatic arthritis. The cyst was located in the medial zone [14] of the tibial component two years postoperatively (Figure 5). We observed a posterior osteophyte overhanging distally from the tibial plafond in 3 primary osteoarthritis ankles.

\section{Complications concomitant procedures (within the first year)}

There were 2 intraoperative complications: one ankle sustained a fracture of the lateral malleolus and one ankle a fracture of the medial malleolus (Figure $6)$. The lateral malleolar fracture was treated nonoperatively with a plaster boot in neutral position $\left(90^{\circ}\right.$ of ankle flexion). The medial malleolar fracture underwent internal fixation surgery and then a plaster boot was applied (Figure 7). Complete healing occurred in 35 days in the first case and in $\mathbf{3 0}$ days in the second one.

A total of 8 concomitant procedures were performed in 5 patients: 2 subtalar arthrodesis, 2 Achilles tendon lengthening, 3 malleolar osteotomies (1 lateral and 2 medial osteotomies), and 1 revision of the talar component for a presumable oversizing.

Complications and reoperations were significantly more frequent in patients with primary osteoarthritis. Delayed wound healing occurred in 2 ankles ( 2 patients, 1 man and 1 woman), with primary osteoarthritis. These patients were treated with weekly medications and the complete wound healing was achieved in 30 and 66 days, respectively. In our series there were no cases of infection or aseptic loosening of prosthetic components.

\section{Discussion}

Despite ankle arthrodesis initially showing good results in the treatment of TT arthritis, the long-term follow-ups revealed potential shortcomings of this treatment, with secondary suffering and degeneration of adjacent joints, altered gait characteristics, and functional limitations [15].

The interest in TAR has been renewed for reasons such as the risk in secondary adjacent hindfoot arthritis after ankle arthrodesis, the desire to relieve pain, and maintain preoperative ankle ROM [16].

The true TT ROM for ankle arthroplasty is determined best by radiography [17]. Since the ankle ROM is a combination of TT and midfoot motion, Coetzee, et al. demonstrated that the radiographic measurement 

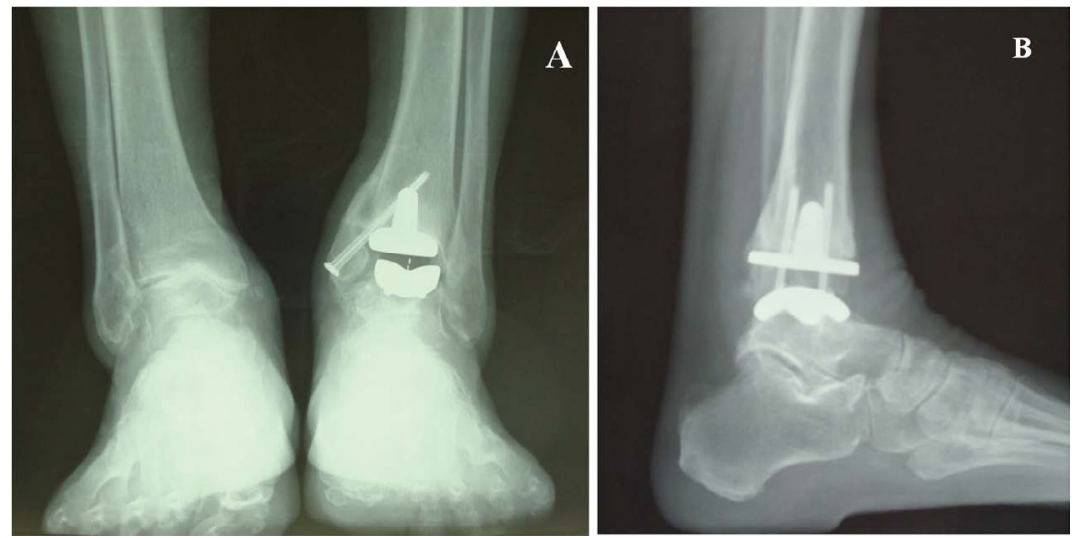

Figure 7: Unstable fracture of the medial malleolus. Postsurgical radiographs of internal fixation with screws A) AP view; B) Lateral view.
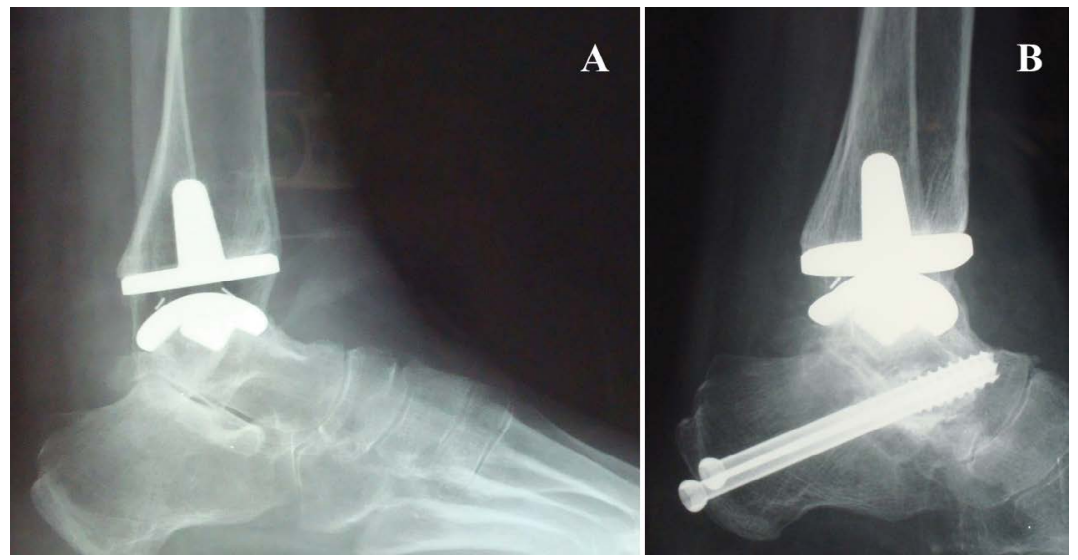

Figure 8: A) Subtalar joint osteoarthritis onset 1 year after TAR; B) Subtalar arthrodesis, iliac allograft, 2 screws. No cyst, no radiolucency, no subsidence.

represents the only reproducible manner to quantify the true ROM of the TT joint after TAR. Clinical measurements are not valid for measuring the true mobility in the ankle joint as they are influenced by the neighboring joints [13].

Although there is no uniformity of results, previous studies have suggested that the maximal improvement in ankle ROM occurs within the first 6 months after surgery [5]. Our results are in agreement with these data. However, the overall improvement, expressed in terms of patient's global satisfaction, was obtained within the first year. For this reason, TAR can be performed as a single surgical procedure only on the TT joint when there is an isolated ankle arthritis. If other pathological conditions, involving neighboring joints coexist, concomitant surgical procedures must be performed.

Wood, et al. defined the following additional procedures: reattachment of the anterolateral capsule, gastrocnemius recession, talonavicular fusion, calcaneal osteotomy and osteotomy of the first metatarsal [14]. Additional procedures can be performed both during the implant of TAR, if needed (contemporary additional procedures), or later, in relation to the clinical situation (subsequent additional procedures). In our series we preferred not to carry out contemporary procedures, instead performing a reoperation after the full recovery of ankle ROM and the eventual onset of pain or other disorders not previously manifested (Figure 8). It is essential to plan not only the possible associated surgical acts but also the time interval in which to achieve the second procedure.

Reoperations include all additional procedures not performed during the implantation of the prosthesis. They can involve: the soft tissues, the bone (osteophytes resection, malleolar osteotomy), or the polyethylene substitution (without prosthetic revision). On the contrary, revisions interest the bone-implant interface and represent the failure of primary implant $[18,19]$. In 1 ankle a revision and substitution of the talar component was necessary for a presumable oversizing. However, the bony integration was complete.

Bonnin, et al. [20] consider the preoperative CT-scan a basic exam to ensure where osteophytes and bone fragments are precisely located. We believe that, if necessary, CT-scan can represent a valid postoperative examination to assess medium-term bony integration of the implant, the presence of cysts and the effective joint space. Indeed, as Viste, et al. [21] sustain, plain radiographs underestimate the presence and extent 
of lesions and CT-scan exam is more sensitive than conventional X-rays to detect osteolysis. However, even if the presence of periprosthetic bone cysts does not represent a sign of loosening and it is not necessary to perform secondary surgery to graft tibial cysts, it determines an extension of the effective joint space. This space includes all periprosthetic regions and is accessible to joint fluid, thus accessible to particulate debris $[20,22]$.

Postoperative radiography represents the best method to assess prosthetic alignment, but this is not the gold standard to evaluate the presence of: small cysts, resorption areas, or posterior osteophytes that are asymptomatic. Therefore, the radiographic evaluation must be associated to pre- and post-operative clinical examination.

Rippstein, et al. [8] distinguish the angle $\alpha$ between the axis of the tibial component stem and the axis of the tibia (an angle of $>5$ was defined as varus or valgus alignment of the tibial component) from the $\beta$ angle that measures the posterior slope of the tibial component with respect to the tibial axis on the lateral radiograph. In our study all the arthroplasty components were correctly centered in the frontal plane, while there were 6 ankles with a posterior slope ( $\beta$ angle) $>5^{\circ}$. No malalignments between the axis of the tibial and the talar components was observed.

The posterior region can be assessed for the presence of posterior osteophytes. They seem to be highest in patients with primary ankle arthritis and lowest in patients with posttraumatic and rheumatoid arthritis. Bone overgrowth, however, never induced symptomatic impingement nor reduction in ankle ROM.

Although that rehabilitation represents an integral part of the TAR intervention, there are few studies that focus on the postoperative management after TAR. The rehabilitation program is divided in two phases: pre- and post-operative. The clinical evaluation should be a combined collaboration by the orthopedist and physiatrist ("binary" evaluation), in order to evaluate the foot support and gait cycle preoperatively. Moreover, patient's factors such as associated deformities, peripherical vascular diseases, concomitant skin disorders, neuropathy, and other conditions that may affect the results must be considered; even if not directly involving bony structures.

In the preoperative evaluation the physician has to consider patient's general condition to define the objectives, the time required to achieve them and develop a rehabilitation protocol tailored to patient's individual need.

So far, there are no scientific guidelines concerning the optimal duration of postsurgical immobilization and thus, there is large variation in these parameters between studies. Most surgeons follow a conservative approach recommending weight bearing after 4-6 weeks after surgery [23]. We believe that third generation implants, if components are well positioned, allow to support early weight bearing in safety, without damaging bony integration. Moreover, early weight bearing may prevent postoperative ankle stiffness due to equines contracture and improve ankle joint ROM. Early partial weight-bearing is a reasonable approach which facilitates bone ongrowth. This is the reason why we recommend an early weight-bearing with the aid of two Canadian crutches, as tolerated by the patient, in the first six weeks. In this period, in patients with osteoporotic bone and/or those who have undergone additional procedures such as corrective osteotomy, 15 kg partial weight-bearing can be allowed.

Postoperative ankle motion reeducation is facilitated by the execution of passive and active ankle mobilization in the prone position, in order to have the maximum global relaxation of the patient and particularly of the muscles of the posterior region of the leg. This method can be switched to the supine position with $90^{\circ}$ flexed knee, which, however, is less stable and requires greater control by the patient.

During the postoperative care, proprioception training is effective in improving postural control, static, and dynamic balance by stimulating afferent inputs. The underlying neural adaptations were shown to occur at different sites of the central nervous system and the plasticity of the spinal, corticospinal, and cortical pathways proved to be highly task specific. For this reason, early neuromotor training is considered the preferable treatment strategy $[24,25]$.

Functional task practice and repetition are key elements that emphasize the plasticity of the sensorimotor system, particularly the spinal and supraspinal structures. In this way a limited and awkward motor sequence is gradually converted into fluid action at a higher speed, with an increased strength and improved reaction time. This training induces adaptations in all the sensory systems assisting postural control, such as the vestibular, the visual, and the somatosensory system as well as in the motor systems controlling muscular output. The exposure to unstable support surfaces provides the activation of proprioceptive system with beneficial in training postural control [26].

The introduction of low-impact physical activity can take place 6 months after surgery and can represent itself a part of the rehabilitation program. Hydrotherapy and swimming can allow the body image pattern rescue and the re-training of the normal gait cycle.

The TAR is a surgical procedure that requires a strong pre and postoperative collaboration between the patient, the surgeon and the physiatrist.

The high rate of patient satisfaction after TAR is likely due to decreased pain, while observing some increase, 
but less than expected, in ROM. This result may be explained by the anterior surgical access, relatively large compared to the narrow ankle circumference, the amount of vascular structures and tendons, and the movement limiting effects of the surgical scar.

Our series demonstrated the best results when compared with previous studies. These findings can be explained by the close cooperation between orthopedist and physiatrist and by anticipating weight bearing and reeducation times.

\section{Conflicts of Interest Statement}

The authors whose names are listed immediately below certify that they have NO affiliations with or involvement in any organization or entity with any financial interest (such as honoraria; educational grants; participation in speakers' bureaus; membership, employment, consultancies, stock ownership, or other equity interest; and expert testimony or patentlicensing arrangements), or non-financial interest (such as personal or professional relationships, affiliations, knowledge or beliefs) in the subject matter or materials discussed in this manuscript.

\section{References}

1. Easley ME, Adams SB Jr, Hembree WC, DeOrio JK (2011) Results of total ankle arthroplasty. J Bone Joint Surg Am 93: 1455-1468.

2. Kofoed H (2006) Some reflections about the evolution of ankle prosthesis. Foot and ankle in rheumatoid arthritis 201-206.

3. Wukich DK, Tuason DA (2011) Diagnosis and treatment of chronic ankle pain. Instr Course Lect 60: 335-350.

4. Barg A, Pagenstert GI, Hügle T, Gloyer M, Wiewiorski M, et al. (2013) Ankle osteoarthritis: Etiology, diagnostics, and classification. Foot Ankle Clin 18: 411-426.

5. Ajis A, Henriquez $H$, Myerson M (2013) Postoperative range of motion trends following total ankle arthroplasty. Foot Ankle Int 34: 645-656.

6. Smith CL (1980) Physical therapy management of patients with total ankle replacement. Phys Ther 60: 303-306.

7. Deorio JK, Easley ME (2008) Total ankle arthroplasty. Instr Course Lect 57: 383-413.

8. Rippstein P, Huber M, Coetzee C, Naal F (2011) Total ankle replacement with use of a new three-component implant. J Bone Joint Surg Am 93: 1426-1435.

9. Hintermann B, Valderrabano V, Dereymaeker G, Dick W (2004) The Hintegra ankle: Rationale and short-term results of 122 consecutive ankles. Clin Orthop Relat Res 57-68.

10. Krause FG, Di Silvestro M, Penner MJ, Wing KJ, Glazebrook MA, et al. (2010) Inter- and intraobserver reliability of the COFAS end-stage ankle arthritis classification system. Foot Ankle Int 31: 103-108.

11. Haskell A, Mann RA (2004) Ankle arthroplasty with preoperative coronal plane deformity: Short-term results. Clin Orthop Relat Res 98-103.

12. Kitaoka HB, Alexander IJ, Adelaar RS, Nunley JA, Myerson MS, et al. (1994) Clinical rating systems for the ankle- hindfoot, midfoot, hallux, and lesser toes. Foot Ankle Int 15: 349-353.

13. Coetzee C, Castro MD (2004) Accurate measurement of ankle range of motion after total ankle arthroplasty. Clinical Orthop Relat Res 27-31.

14. Wood PLR, Karski MT, Watmough P (2010) Total ankle replacement: The results of 100 mobility total ankle replacements. J Bone Joint Surg Br 92: 958-962.

15. Sproule JA, Chin T, Amin A, Daniels T, Younger AS, et al. (2013) Clinical and radiographic outcomes of the mobility total ankle arthroplasty system: Early results from a prospective multicenter study. Foot Ankle Int 34: 491-497.

16. Kopp FJ, Patel MM, Deland JT, O'Malley MJ (2006) Total ankle arthroplasty with the agility prosthesis: Clinical and radiographic evaluation. Foot Ankle Int 27: 97-103.

17. Elgaard T, Kofoed H (1999) Radiographic assessment of the mobility of ankle arthroplasty: A prospective study. Foot and Ankle Surgery 5: 95-99.

18. Henricson A, Nilsson J, Carlsson A (2011) 10-year survival of total ankle arthroplasties. A report on 780 cases from the swedish ankle register. Acta Orthop 82: 655-659.

19. Zaidi R, Cro S, Gurusamy K, Siva N, Macgregor A, et al. (2013) The outcome of total ankle replacement: A systematic review and meta-analysis. Bone Joint J 95: 1500-1507.

20. Bonnin M, Gaudot F, Laurent JR, Ellis S, Colombier JA, et al. (2011) The Salto total ankle arthroplasty: Survivorship and analysis of failures at 7 to 11 years. Clin Orthop Relat Res 469: 225-236.

21. Viste A, Al Zahrani N, Brito N, Lienhart C, Fessy MH, et al. (2015) Periprosthetic osteolysis after AES total ankle replacement: Conventional radiography versus CT-scan. Foot Ankle Surg 21: 164-170.

22. Schmalzried TP, Jasty M, Harris WH (1992) Periprosthetic bone loss in total hip arthroplasty. Polyethylene wear debris and the concept of the effective joint space. J Bone Joint Surg Am 74: 849-863.

23. Schutte BG, Louwerens JWK (2008) Short-term results of our first 49 Scandinavian total ankle replacement (STAR). Foot Ankle Int 29: 124-127.

24. Elliott D, Grierson LE, Hayes SJ, Lyons J (2011) Action representations in perception, motor control and learning: Implications for medical education. Med Educ 45: 119-131.

25. Brustio PR, Magistro D, Ivaldi S, Caglio MM, Rabaglietti E, et al. (2015) Neuromotor training in older women living in long-term care setting: A pilot study. Geriatr Nurs 36: 361366.

26. Karakaya MG, Rutbll H, Akpinar E, Yildirim A, Karakaya IÇ (2015) Effect of ankle proprioceptive training on static body balance. J Phys Ther Sci 27: 3299-3302. 